\title{
Broad-angle negative reflection and focusing of elastic waves from a plate edge
}

\author{
Istvan A. Veres, ${ }^{1}$ Clemens Grünsteidl, ${ }^{1}$ David M. Stobbe,${ }^{2}$ and Todd W. Murray ${ }^{2}$ \\ ${ }^{1}$ Research Center for Non-destructive Testing GMBH (RECENDT), Altenberger Strasse 69, 4040 Linz, Austria \\ ${ }^{2}$ Department of Mechanical Engineering, University of Colorado at Boulder, Boulder, Colorado 80309, USA
}

(Received 28 January 2016; revised manuscript received 18 March 2016; published 4 May 2016)

\begin{abstract}
Guided elastic waves in plates, or Lamb waves, generally undergo reflection and mode conversion upon encountering a free edge. In the case where a backward-propagating Lamb wave is mode-converted to a forwardpropagating wave or vice versa, the mode-converted wave is reflected on the same side of the surface normal as the incident wave. In this paper, we study such negative reflection and show that this effect can be achieved over a broad angular range at a simple plate edge. We demonstrate, through both numerical and experimental approaches, that a plate edge can act as a lens and focus a mode-converted Lamb wave field. Furthermore, we show that as the wave vectors of the incident and mode-converted Lamb waves approach each other, the mode-converted field nearly retraces the incident field. We propose that broad-angle negative reflection may find application in the nondestructive testing of structures supporting guided waves and in the development of new acoustic devices including resonators, lenses, and filters.
\end{abstract}

DOI: 10.1103/PhysRevB.93.174304

\section{INTRODUCTION}

In both the optics and acoustics communities, there has been significant interest in developing new approaches for manipulating wave fields using backward-propagating waves. Backward wave propagation can be defined as that in which the direction of the group velocity is opposite to that of the phase velocity, and thus the wave crests and troughs appear to propagate against the direction of energy flow. The existence of such exotic behavior was first deliberated by Lamb in the early 20th century [1]. Designer materials that incorporate subwavelength resonant structures (optical and acoustic metamaterials) or wavelength-scale periodicity (photonic and phononic crystals) can support backward waves, and a variety of nonintuitive physical effects such as negative refraction and subwavelength focusing have been demonstrated [2-13]. It is also well known that optical and acoustic waveguides support backward wave propagation [14-18]. Elastic waves propagating in plates, cylinders, rods, and supported layers, for example, all show backward wave propagation over some region of frequency and wave number space [15-19].

While the existence of backward waves in basic elastic structures is well known, it is only recently that related phenomena have been used to characterize materials and manipulate guided wave fields. Zero-group-velocity (ZGV) resonances in thin plates and cylinders, for example, occur at specific points within the dispersion relation where the group velocity goes to zero while the phase velocity remains finite [15]. The physical origin of these resonances is the interference between forward- and backward-propagating waves with the same wave number. Sharp resonances are observed at ZGV frequencies, and these have been used for the measurement of material properties and dimensions,

Published by the American Physical Society under the terms of the Creative Commons Attribution 3.0 License. Further distribution of this work must maintain attribution to the author(s) and the published article's title, journal citation, and DOI. defect detection, and adhesion testing [20-24]. In the case of Lamb waves in plates, it was also demonstrated that mode conversion between backward-propagating and forwardpropagating waves (or vice versa) at a step change in thickness leads to negative refraction, a process in which the refracted ray leaves the interface at an angle that is on the opposite side of the interface normal to that predicted by the usual Snell's law $[25,26]$. A Lamb wave lens was designed by incorporating a simple thickness trough in an otherwise homogeneous plate. Forward-propagating waves emitted from the source position were mode converted to backward-propagating waves at the first trough interface, and underwent negative refraction and focusing within the lens. Upon encountering the second trough interface, the waves were mode converted back to forwardpropagating waves and again underwent negative refraction and focusing on the distal side of the lens. Effective mode conversion was observed over a broad range of incident angles, producing a lens with a high numerical aperture.

Negative reflection of guided elastic waves is an intriguing phenomenon in which a reflected wave is directed on the same side of the surface normal as the incident wave [27]. Negative reflection at an interface is expected to occur when a backward wave is mode converted to a forward wave upon reflection from an interface (and vice versa). Negative reflection of Lamb waves was demonstrated by Germano et al. by sending a forward-propagating wave to the free edge of a homogeneous, isotropic, steel plate and observing that the reflected field fell on the same side of the surface normal as the incident wave, and the result was confirmed at two different angles of incidence [27]. In this paper, we further explore negative reflection at the free edge of a plate and show that efficient negative reflection can be achieved over a broad angular range at a simple plate edge. We demonstrate focusing of a Lamb wave field upon reflection from a plate edge and show that near the zero-group-velocity point, where the magnitudes of the wave vectors of forward- and backward-propagating waves are similar, the reflected field nearly retraces the incident field. The plate edge acts similarly to a phase-conjugate mirror [28]. Negative reflection could have important implications in the 
(a)

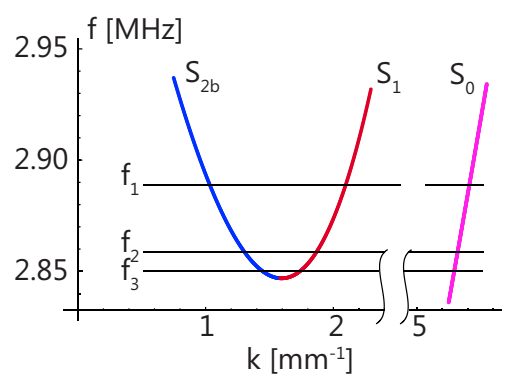

(b)

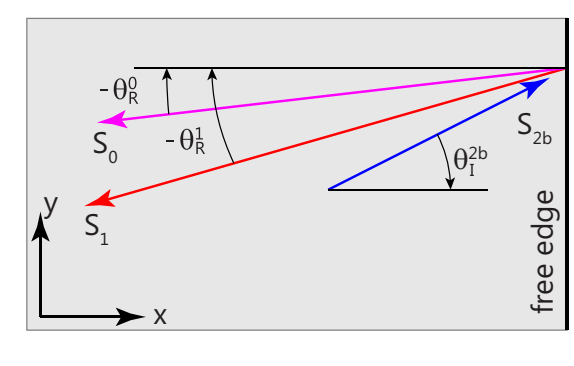

(c)

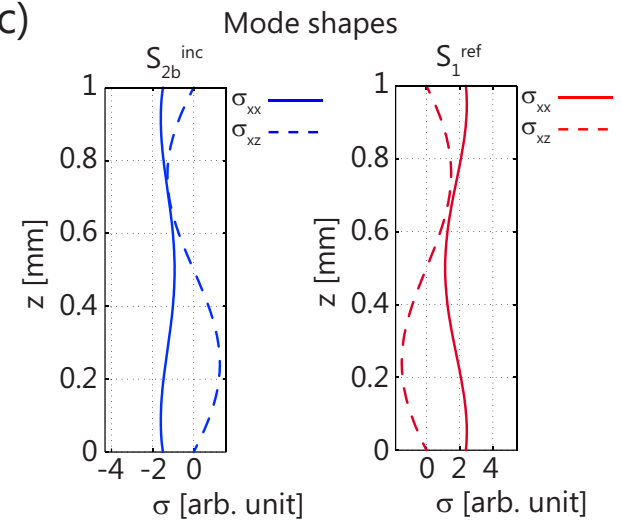

FIG. 1. (a) Dispersion curve for symmetric Lamb waves in the vicinity of the ZGV point for a 1.0-mm-thick aluminum plate. (b) Mode conversion of an incident $S_{2 b}$ mode at a free edge leads to negative reflection of the $S_{0}$ and $S_{1}$ modes. $\theta_{I}$ is the angle of incidence and $\theta_{R}$ is the angle of reflection. (c) The mode shapes of the incident $S_{2 b}$ and the reflected $S_{1}$ modes near the ZGV point at a frequency of $2.85 \mathrm{MHz}$.

nondestructive testing of structures supporting guided waves, and in the development of new acoustic devices.

\section{NEGATIVE REFLECTION}

Here we consider negative reflection in a homogeneous, isotropic aluminum plate with a $1.0 \mathrm{~mm}$ thickness and longitudinal and transverse wave velocities of $6.31 \mathrm{~mm} / \mu \mathrm{s}$ and $3.11 \mathrm{~mm} / \mu \mathrm{s}$, respectively. Lamb wave propagation in the plate is described by the Rayleigh-Lamb equation, which provides a relationship between the frequency and wave vector of the supported modes [29]. At a given frequency, a finite number of propagating modes exist, along with nonpropagating (evanescent) modes. Furthermore, the modes are classified as symmetric or antisymmetric depending on the symmetry of the displacement field produced with respect to the mid-plane of the plate. For our purposes, it is sufficient to consider the dispersion curve over the small frequency range shown in Fig. 1(a). Here, only the real, symmetric modes are plotted over positive $k$ values, thus limiting the modes to the $S_{0}, S_{1}$, and $S_{2 b}$. The phase velocity can be found by dividing the ordinate by the abscissa, while the group velocity is given by the slope. At the intersection of the $S_{2 b}$ and $S_{1}$ modes, the group velocity vanishes. The mode labeled $S_{2 b}$ has a negative group velocity while the other two modes have a positive group velocity; all modes have a positive phase velocity. Note that the dispersion curve is symmetric about $k=0$ such that for $k<0$ the $S_{2 b}$ mode has a positive group velocity while the $S_{0}$ and $S_{1}$ modes have a negative group velocity; all modes have a negative phase velocity in this case.

Now consider the situation shown in Fig. 1(b), where an $S_{2 b}$ Lamb wave is generated at some point in the plate and impinges upon a free edge. Considering that the wave propagates from the source to the edge, it has a positive group velocity and negative phase velocity, or negative $k$. The traction-free boundary condition can be satisfied only if the tangential component of the wave vector is conserved. Thus the angle of reflection $\left(\theta_{R}\right)$ is given by $\theta_{R}=\sin ^{-1}\left[\left(k_{I} / k_{R}\right) \sin \left(\theta_{I}\right)\right]$, where $k_{i}$ and $k_{R}$ are the wave vectors of the incident and reflected waves and $\theta_{I}$ is the angle of incidence. It follows that if a backward-propagating Lamb mode converts to a forwardpropagating mode at a free edge, the angle of reflection must be negative. Also, in the limiting case that the magnitude of the wave vectors of the incident and reflected waves are equal, then the reflected wave will retrace the incident wave and propagate back to the source.

Lamb wave mode conversion at a free edge for both normal [30-32] and oblique [33-35] incidence has received considerable attention in the literature. Considering a normally incident plane wave and referring back to Fig. 1(b), the boundary condition that must be satisfied is that the normal $\left(\sigma_{x x}\right)$ and shear $\left(\sigma_{x z}\right)$ traction on the edge surface must vanish. These stresses, in turn, are the sum of the stresses produced by the incident and reflected waves. In general, it is not possible for the boundary condition to be satisfied by a single Lamb mode, because the shear stress switches sign upon reflection while the normal stress does not [30]. Summing the incident and reflected waves at the boundary can therefore not satisfy the boundary condition, regardless of the phase of the reflected wave. In general, one must consider the relative contributions of all propagating and nonpropagating modes at a particular frequency that will sum to satisfy the boundary condition at a given angle of incidence. Forward and backward Lamb waves near the $\mathrm{ZGV}$ point, however, show a somewhat unique behavior. In this region of the dispersion curve, it has been shown that a normally incident $S_{2 b}$ wave is mode converted upon reflection from a free edge to a forward-propagating $S_{1}$ mode with a reflection coefficient near unity [33]. The opposite effect is also predicted for a normally incident $S_{1}$ mode, which mode converts with exceptional efficiency to a backward-propagating $S_{2 b}$ mode [33]. In order to understand why this is the case, we can consider the stress mode shapes shown in Fig. 1(c) at a frequency of $2.85 \mathrm{MHz}$, with the mode shape of the reflected $S_{1}$ wave shown with a $\pi$ phase shift with respect to the incident $S_{2 b}$. It is clear that the stress fields are very nearly opposite such that the sum of the stresses at the free edge can satisfy the boundary condition. It is important to emphasize that this behavior is not often observed, and stems from the fact that the mode shapes of the $S_{1}$ and $S_{2 b}$ modes near the $\mathrm{ZGV}$ point are very similar but the (slow) group velocities for the modes are in opposite directions. 
(a)

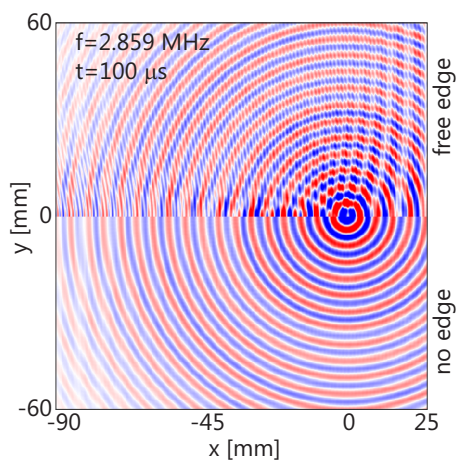

(b)

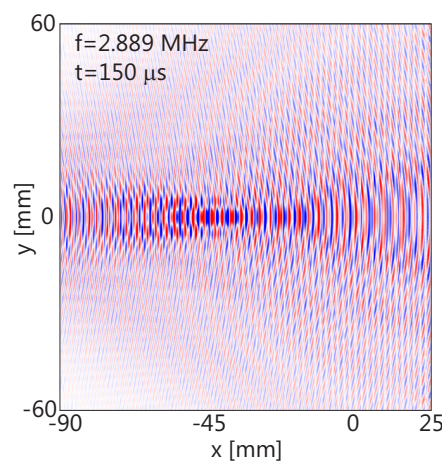

(c)

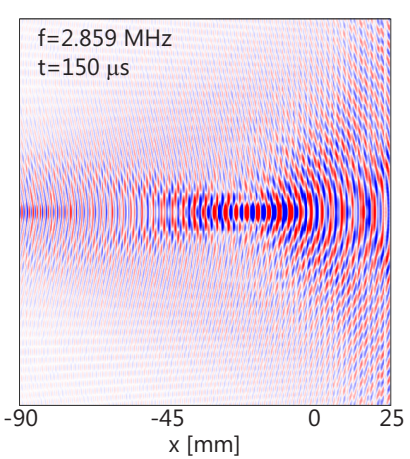

(d)

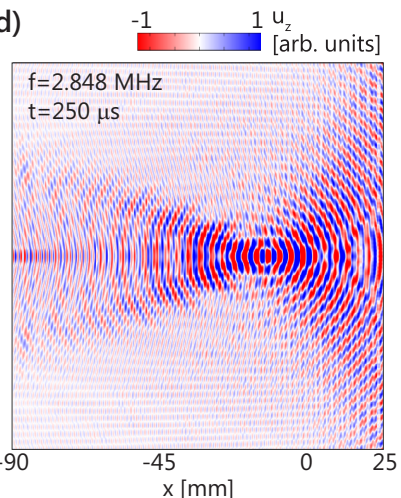

FIG. 2. (a) Simulated out-of-plane surface displacements of the plate in the presence of a free edge at $x=25 \mathrm{~mm}$ (top, $y>0$ ) and in the absence of a free edge (bottom, $y<0$ ) for $f=2.859 \mathrm{MHz}$ at $t=100 \mu \mathrm{s}$. (b)-(d) Reflected wave fields from the free edge at (b) $f=2.889 \mathrm{MHz}$ and $t=150 \mu \mathrm{s}$, (c) $f=2.859 \mathrm{MHz}$ and $t=150 \mu \mathrm{s}$, and (d) $f=2.848 \mathrm{MHz}$ and $t=250 \mu \mathrm{s}$. The later time in (d) accounts for the slow group velocity in the close vicinity of the $\mathrm{ZGV}$ point.

\section{NUMERICAL SIMULATION}

Negative reflection from a plate edge was simulated with the finite-element time-domain method using a commercial software package (PZFLEX, Weidlinger Associates, Mountain View, CA). We consider an aluminum substrate with a surface extending over $115 \times 180 \mathrm{~mm}^{2}$ and a thickness of $1.0 \mathrm{~mm}$. Only one-quarter of the plate is modeled by employing symmetric boundary conditions at the edge of the plate ( $y=0 \mathrm{~mm}$ in Fig. 2) and at the plate bottom $(z=0.5 \mathrm{~mm})$. The symmetric boundary condition at the bottom of the plate suppresses the generation of antisymmetric modes, but a simulation with a full-thickness plate showed that these modes have negligible contribution to the generated and reflected wave fields. We consider the response of the plate to a laser source, with the surface forcing function represented by a simplified distributed dipole model consisting of a radially directed in-plane force with a spatial distribution given by $f(r)=r e^{-r^{2} R d^{2}}$, where $d=1.51 \mathrm{~mm}$, giving a full width at half maximum of $3.55 \mathrm{~mm}$. The forcing function is sinusoidal in time. The sample is modeled on an orthogonal grid with element dimensions of $47 \times 45 \times 45 \mu \mathrm{m}^{3}(x, y, z)$. The source is located $25 \mathrm{~mm}$ from the plate edge at the origin of the coordinate system.

Figure 2(a) shows two out-of-plane displacement fields calculated over the plate surface at a frequency of $2.859 \mathrm{MHz}$ [ $f_{2}$ in Fig. 1(a)] and a time of $100 \mu$ s. The top part of the image $(y>0 \mathrm{~mm})$ shows the displacement field with the free edge at $x=25 \mathrm{~mm}$. The bottom image $(y<0 \mathrm{~mm})$ shows the incident displacement field in the absence of a free edge, where the plate was extended and terminated with absorbing boundary conditions. In both cases, the dominant mode seen propagating out from the source position is the $S_{2 b}$ mode, with a wavelength of $5.0 \mathrm{~mm}$. The reason for this is that the excitation spot size is larger than the wavelengths of the $S_{0}$ and $S_{1}$ modes and therefore does not couple well into these modes. The additional higher spatial frequency structure observed when the free edge is present is the result of reflection and mode conversion at the interface. In order to observe the reflected field more clearly, we calculated the displacement fields with a free edge and the incident field without an edge at three frequencies shown on Fig. 1(a) as $f_{1}-f_{3}$. At each frequency, the incident field was subtracted from the field with the free edge, leaving only displacement components associated with the reflected field. The results for the three frequencies are shown in Figs. 2(b)-2(d). Interestingly, there is very little evidence of a reflected and diverging $S_{2 b}$ mode but rather strong mode conversion into the forward-propagating $S_{1}$ mode. Negative reflection and associated focusing are observed, with the focal volume translating back toward the source as the frequency is decreased towards the ZGV point and the magnitudes of the wave vectors for the $S_{1}$ and $S_{2 b}$ modes approach each other. Broad-angle negative reflection is particularly evident at the lowest frequency, where the reflected wave field focuses just behind the source position. Negative reflection and focusing of the $S_{0}$ mode can also be seen at the extreme left of the images along $y=0 \mathrm{~mm}$. This effect is not as pronounced as with the $S_{1}$ mode due to the large difference in $k$ vectors and less efficient mode conversion. Finally, we note that the simulations were run to allow sufficient time for the reflected $S_{1}$ wave field to fully develop through the focal volume. Due to the low group velocity, the $S_{1}$ wave field did not fully develop over the entire simulated region, leading to the weaker field after the focus seen in the plots.

Further insight into negative reflection can be obtained by examining the field in Fourier space. For each simulation frequency, the time trace from each spatial position across the plate was collected. The last $100 \mu$ s of each temporal wave form was then Fourier transformed and the complex value of the fast Fourier transform (FFT) at the excitation frequency determined. This array was processed using a two-dimensional (spatial) Fourier transform, yielding the magnitude plots shown in Figs. 3(a)-3(c). The lowest spatial frequency ring in these plots $\left(|k|\right.$ between 1.0 and $\left.1.4 \mathrm{~mm}^{-1}\right)$ corresponds to the $S_{2 b}$ mode which propagates at all angles from the source. The $S_{1}$ mode, with the next higher spatial frequency ( $|k|$ between 1.8 and $2.2 \mathrm{~mm}^{-1}$ ) exists over a more narrow angular range corresponding to the possible angles of negative reflection. Given that the largest possible angle of incidence is $90^{\circ}$, the largest possible angle of reflection is given by $\theta_{m}=\sin ^{-1}\left(k_{i} / k_{R}\right)$. For the $S_{1}$ mode, this gives a $\theta_{m}$ of $28.3^{\circ}$, $40.1^{\circ}$, and $47.3^{\circ}$ for $f_{1}, f_{2}$, and $f_{3}$, respectively. These angles 
(a)

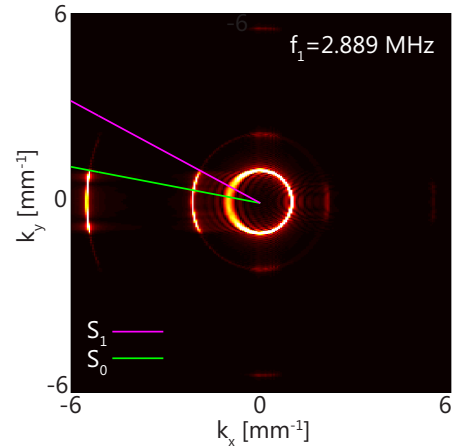

(b)

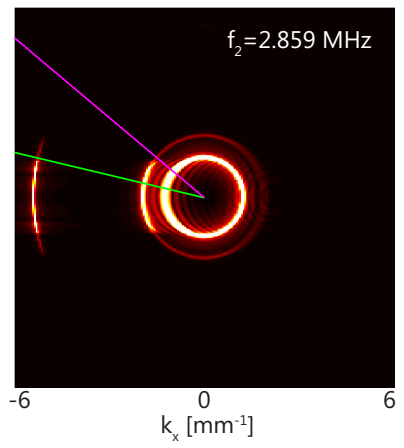

(c)

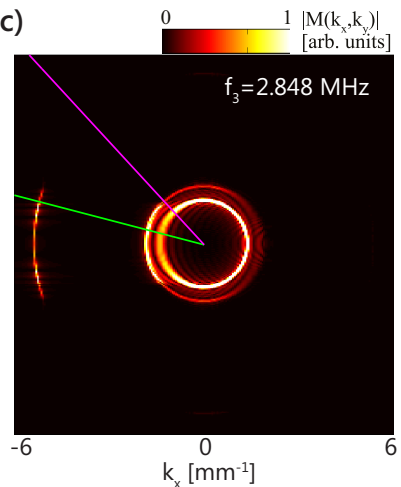

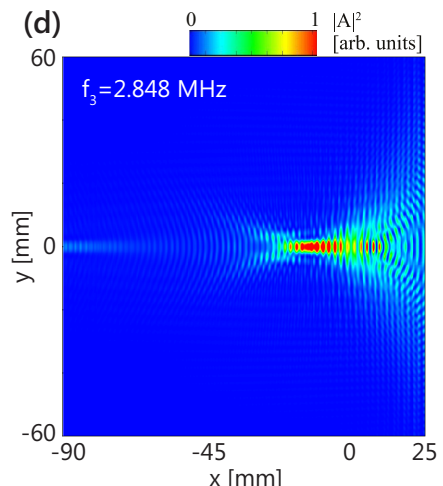

FIG. 3. Fourier domain representation of the wave fields at the frequencies of (a) $2.889 \mathrm{MHz}$, (b) $2.859 \mathrm{MHz}$, and (c) $2.848 \mathrm{MHz}$. The inner ring with the lowest spatial frequency corresponds to the excited $S_{2 b}$ mode, and the outer circular segments represent the reflected $S_{0}$ and $S_{1}$ modes. The green and magenta lines show the maximum possible angles of reflection for the $S_{0}$ and $S_{1}$ modes, respectively. (d) Intensity plot of the reflected field for the lowest frequency $(f=2.848 \mathrm{MHz})$ showing the focusing of the acoustic energy just beyond the excitation point at $x=0 \mathrm{~mm}$.

are shown as the magenta lines on the plot. Note that within the computational model the maximum angle of incidence is $75^{\circ}$, thus further constraining the angular range of reflection. The increase in the angular range of reflection with reduction in frequency towards the ZGV point is clearly observed, as is the uniformity in the magnitude as a function of angle. Using the same argument for the $S_{0}$ mode, $\theta_{m}$ is $10.8^{\circ}, 13.4^{\circ}$, and $14.6^{\circ}$ for $f_{1}, f_{2}$, and $f_{3}$, respectively. Again, we see general agreement in the magnitude plots, with the green line indicating the maximum angle of reflection. Figure 3(d) shows an intensity plot of the reflected field at a frequency of $2.848 \mathrm{MHz}$. Here, the last $100 \mu$ s of the displacement signal at each spatial location was Fourier transformed to determine the magnitude at the excitation frequency, and this was squared to yield the intensity plot. The energy of the negatively reflected $S_{1}$ is concentrated within the focal volume, producing a focal spot with a lateral full width at half maximum of $3.7 \mathrm{~mm}$.

\section{EXPERIMENTAL SETUP}

A basic schematic of the experimental setup is shown in Fig. 4(a). An electroabsorption modulated laser operating at a wavelength of $1550 \mathrm{~nm}$ was used for Lamb wave excitation. The output of the laser was sent to a $1.0 \mathrm{~W}$ erbium-doped fiber amplifier, through a collimator and lens, and to the back surface of the sample. The FWHM of the excitation laser spot on the sample surface was $2.06 \mathrm{~mm}$. The laser output was modulated sinusoidally at the desired frequency using a signal generator. Lamb waves were detected on the opposite surface of the sample using a stabilized Michelson interferometer. The output from the interferometer was fed into an radio frequency lock-in amplifier. The reference signal for the lock in was derived from the signal generator driving the excitation laser using a power splitter. The lock-in time constant was $300 \mathrm{~ms}$ with a roll-off of $18 \mathrm{~dB}$ per octave. The excitation laser optics and sample were mounted on a dual-axis motorized translation
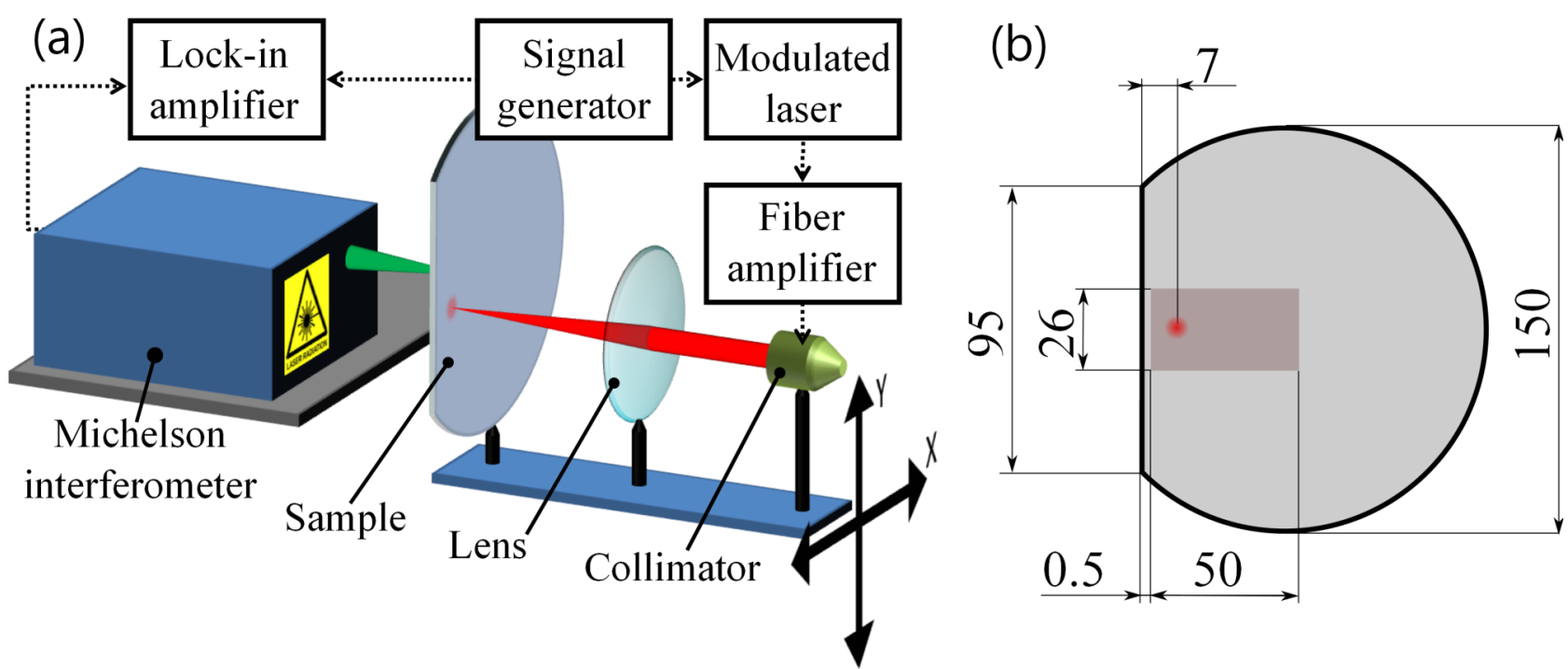

FIG. 4. (a) Schematic of the experimental setup used to excite and detect narrow-bandwidth Lamb waves and (b) the dimensions of the aluminum sample used for the measurements with the excitation point marked by the red dot. 
(a)

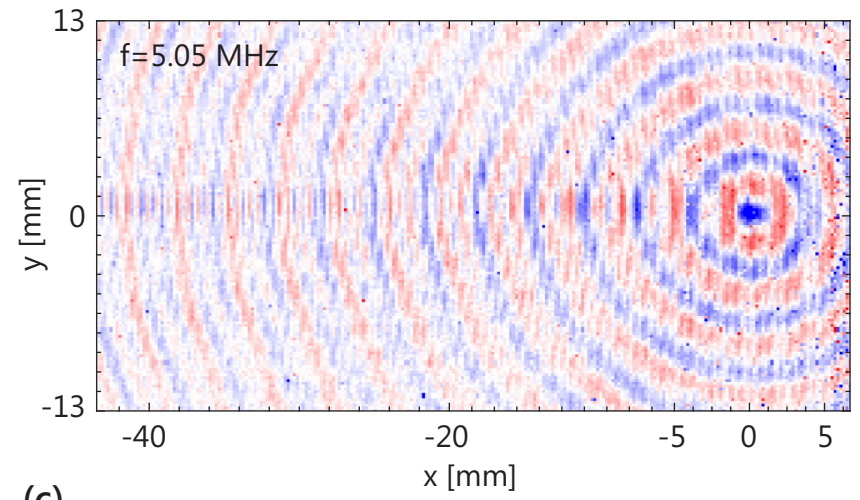

(c)

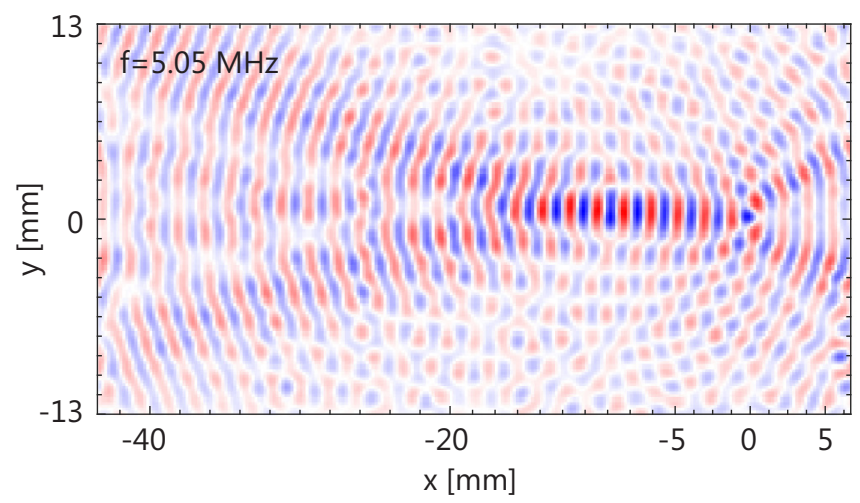

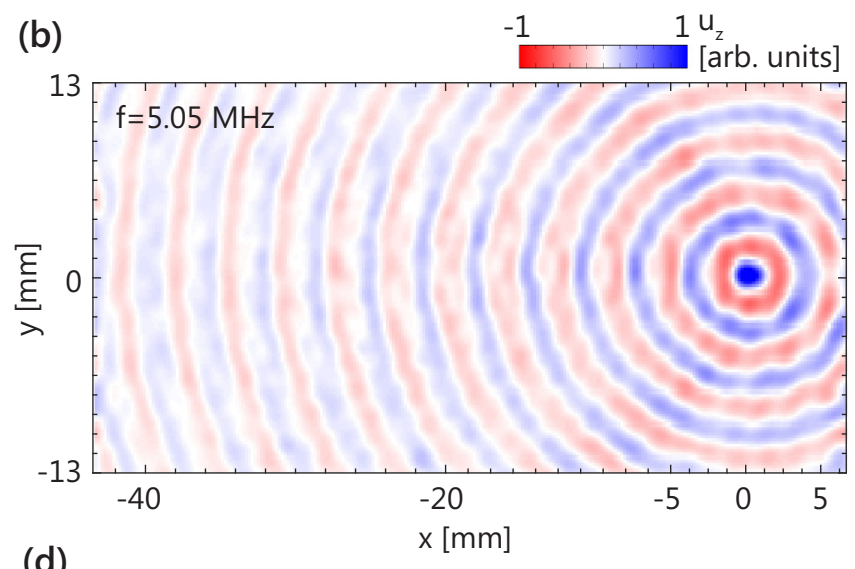

(d)

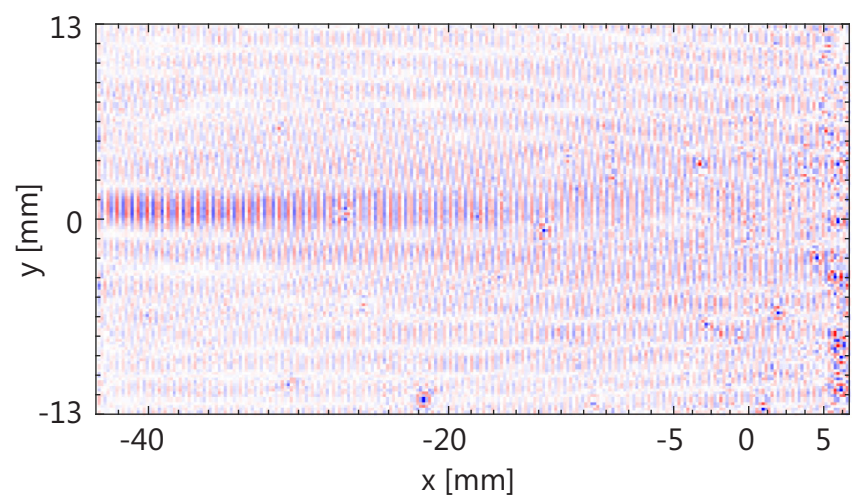

FIG. 5. (a) In-phase component of the raw experimental data at a frequency of $5.05 \mathrm{MHz}$. The wave field is dominated by the $S_{2 b}$ mode with a wavelength of $3.6 \mathrm{~mm}$. (b) Low-pass-filtered data (cutoff $k=3.4 \mathrm{~mm}^{-1}$ ) isolating the $S_{2 b}$ mode. (c) Band-pass-filtered data $\left(k=3.4-4.0 \mathrm{~mm}^{-1}\right.$ ) to isolate the $S_{1}$ mode. Negative reflection and focusing of the field are apparent. (d) Band-pass-filtered data $\left(k=9.2-9.6 \mathrm{~mm}^{-1}\right)$ to isolate the $S_{0}$ mode. Negative reflection leads to a concentration of the field along $y=0 \mathrm{~mm}$ for $x<-20 \mathrm{~mm}$.

stage, allowing for the detection point to be scanned over the sample surface. The excitation point was placed approximately $7.0 \mathrm{~mm}$ from the plate edge and the detection point was scanned over a $50 \times 26 \mathrm{~mm}^{2}$ area with a step size in both dimensions of $0.20 \mathrm{~mm}$. Data were collected at excitation frequencies of 5.000, 5.025, and 5.050 MHz. The sample was an aluminum plate with the dimensions indicated in Fig. 4(b). The ZGV resonance frequency of the plate was measured, by scanning the excitation laser frequency with the source and detector on epicenter, to be $4.980 \mathrm{MHz}$. The thickness of the sample was $578 \mu \mathrm{m}$. The edge of the plate was polished to obtain a $90^{\circ}$ angle with the plate surface. In addition, edges of the plate away from the free edge of interest were covered with adhesive tape to dampen spurious Lamb wave reflections.

\section{EXPERIMENTAL RESULTS AND DISCUSSION}

Figure 5(a) shows the in-phase component of the raw data obtained from the lock-in amplifier at a frequency of $5.05 \mathrm{MHz}$. The source is located at the origin of the coordinate system and the free edge is at $x=7.0 \mathrm{~mm}$, just beyond the scanned region. As in the simulation data, the dominant Lamb wave mode generated is the $S_{2 b}$ mode which has a wavelength of $3.6 \mathrm{~mm}$. There is no apparent interference pattern associated with a reflected $S_{2 b}$ in the raw data. The individual modes can be isolated using a two dimensional spatial frequency filter. Figure 5(b) shows the data low-pass filtered at a spatial frequency of $|k|=3.4 \mathrm{~mm}^{-1}$ isolating only the $S_{2 b}$ mode. Figure 5(c) shows the raw data band-pass filtered between spatial frequencies of $|k|=3.4 \mathrm{~mm}^{-1}$ and $|k|=4.0 \mathrm{~mm}^{-1}$ which isolates the $S_{1}$ mode. Negative reflection of this mode is clearly evident in the experimental data. The phase fronts associated with the $S_{1}$ generated through mode conversion at the edge converge to a cigar-shaped focal area centered near $x=-8 \mathrm{~mm}$. Figure 5(d) shows the raw data band-pass filtered between spatial frequencies of $|k|=9.2 \mathrm{~mm}^{-1}$ and $|k|=9.6 \mathrm{~mm}^{-1}$ which isolates the $S_{0}$ mode. As also seen in the simulations, negative reflection leads to a broad concentration of this mode along the $y=0 \mathrm{~mm}$ axis, particularly for $x<$ $-20 \mathrm{~mm}$.

In order to investigate focusing of the reflected field, the in-phase and quadrature components of the experimental data taken at all three frequencies were band-pass filtered around the spatial frequency of the $S_{1}$ mode. The band-pass filters were applied between spatial frequencies of $3.4-4.0 \mathrm{~mm}^{-1}$, $3.3-4.0 \mathrm{~mm}^{-1}$, and $3.1-4.0 \mathrm{~mm}^{-1}$ for excitation frequencies of $5.050 \mathrm{MHz}, 5.025 \mathrm{MHz}$, and $5.000 \mathrm{MHz}$, respectively. The magnitude squared of the normal surface displacement was then calculated and is shown in Figs. 6(a)-6(c). At all frequencies, the focusing associated with the negative reflection is apparent, with the focus shifting toward the 
(a)

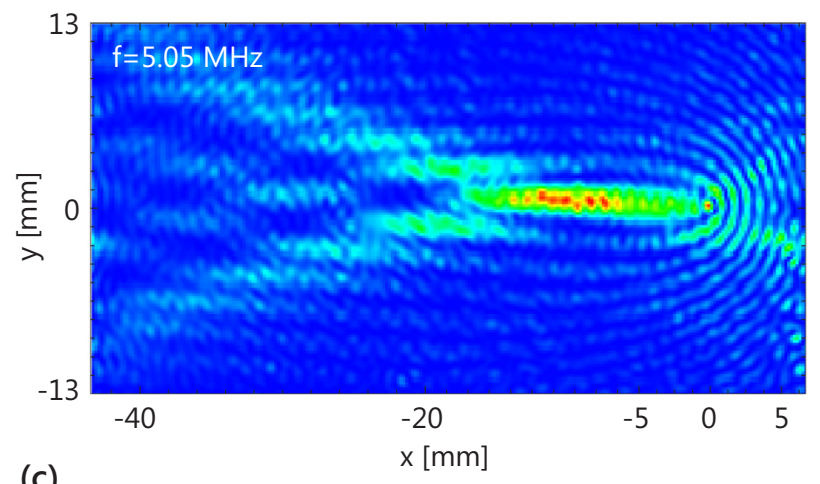

(c)

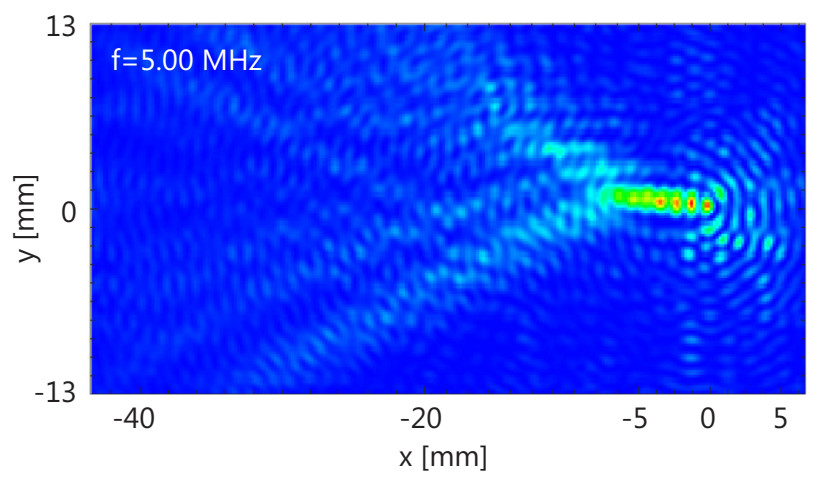

(b) $\quad 0 \quad 1|\mathrm{~A}|^{2}$

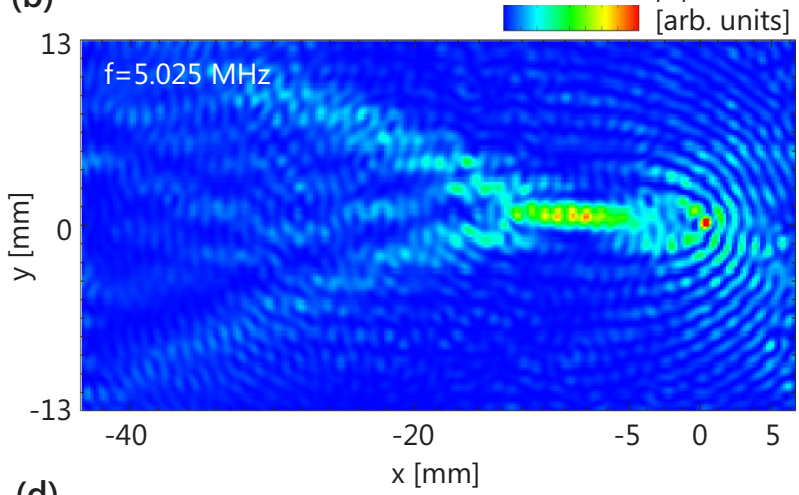

(d)

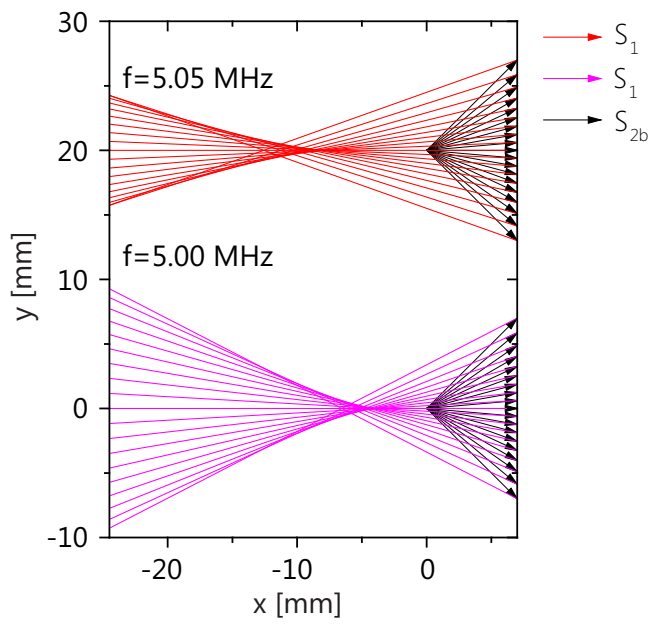

FIG. 6. Squared magnitude of the measured displacement fields at frequencies of (a) 5.050, (b) 5.025, and (c) 5.000 MHz. The data were band-pass filtered to isolate the $S_{1}$ mode with passbands of $k=3.4-4.0 \mathrm{~mm}^{-1}, k=3.3-4.0 \mathrm{~mm}^{-1}$, and $k=3.1-4.0 \mathrm{~mm}^{-1}$ for (a), (b), and (c), respectively. The $S_{1}$ focus recedes towards the excitation point $(x=0)$ as the frequency approaches the ZGV point (d) Ray diagram for negative reflection with mode conversion for 5.000 and $5.050 \mathrm{MHz}$.

excitation point as the magnitudes of the propagation vectors $k$ approach each other near the zero-group-velocity point. Although one must take care to account for the differences in plate geometry and source location, the experimental results are in general agreement with the simulations. In all three cases, some field distortion (beyond that which is seen in the simulations) is observed, which may stem from reflections from additional plate boundaries that were insufficiently damped and small variations in plate thickness over the measurement zone. Nevertheless, broad-angle negative reflection and focusing through mode conversion at a free edge is clearly demonstrated. Figure 6(d) shows a ray diagram which helps in understanding negative reflection with mode conversion where the incident and reflected wave vectors are not the same. Here, we consider the incident and reflected fields at 5.00 and $5.05 \mathrm{MHz}$ (the $5.05 \mathrm{MHz}$ ray diagram is shift up the $y$ axis by $20 \mathrm{~mm}$ for ease of viewing). A source generating the $S_{2 b}$ mode is located at $x=0 \mathrm{~mm}$ and represented by the black arrows. Mode conversion to the $S_{1}$ mode is assumed at a boundary located at $x=7.0 \mathrm{~mm}$. The negative angle of reflection is calculated, using the measured $k$ values, for each incident angle and the reflected rays are shown in the figure. At a frequency of $5.05 \mathrm{MHz}$ we see a convergence of rays associated with a focus over a relatively broad range between $x=-12 \mathrm{~mm}$ and $x=-5 \mathrm{~mm}$ and at $5.00 \mathrm{MHz}$ the focal region shifts toward the source position and is more confined longitudinally to the area between $x=-6 \mathrm{~mm}$ and $x=-2 \mathrm{~mm}$. Comparing these simple ray diagrams to the results shown in Figs. 6(a) and 6(c), we see excellent agreement between the predicted and measured focal regions.

Finally, the angular range of negative reflection can be best visualized using two-dimensional spatial Fourier transforms. The raw experimental data at each frequency were transformed and the resulting magnitude plots are shown in Figs. 7(a)-7(c). As with the simulation data, the lowest spatial frequency ring, with $|k|$ ranging from $1.73 \mathrm{~mm}^{-1}(5.05 \mathrm{MHz})$ to $2.14 \mathrm{~mm}^{-1}$ (5.00 MHz), corresponds to the $S_{2 b}$ mode emitted by the source at all angles. The next higher spatial frequency feature, with $|k|$ values ranging from $3.64 \mathrm{~mm}^{-1}(5.05 \mathrm{MHz})$ to $3.30 \mathrm{~mm}^{-1}$ (5.00 MHz), corresponds to the $S_{1}$ mode, and the highest spatial frequency, with $|k|$ values ranging from $9.41 \mathrm{~mm}^{-1}$ (5.05 MHz) to $9.25 \mathrm{~mm}^{-1}$ (5.00 MHz) corresponds to the $S_{0}$ mode. Following the approach used in the simulation results, one can calculate a maximum angle of reflection $\left(\theta_{m}\right)$ based on the $k$ values of the incident and reflected wave modes. For the $S_{1}$ mode, these angles are $28.4^{\circ}, 32.9^{\circ}$, and $40.5^{\circ}$ for frequencies of $5.05 \mathrm{MHz}, 5.025 \mathrm{MHz}$, and $5.00 \mathrm{MHz}$, respectively. For the $S_{0}$ mode the angles lie between $10.6^{\circ}$ and 


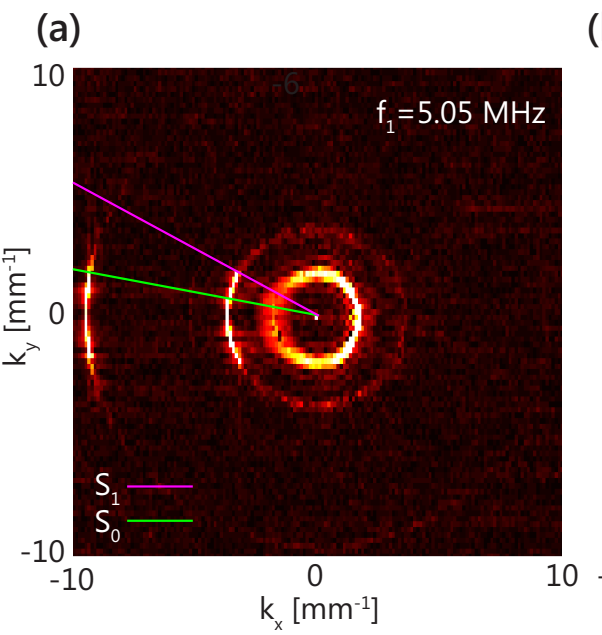

(b)

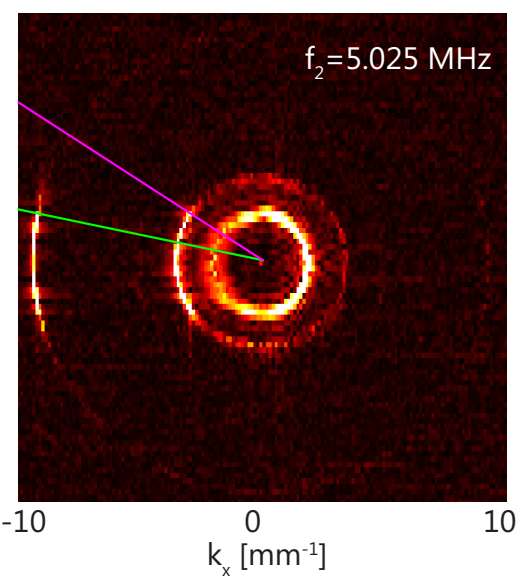

(c)

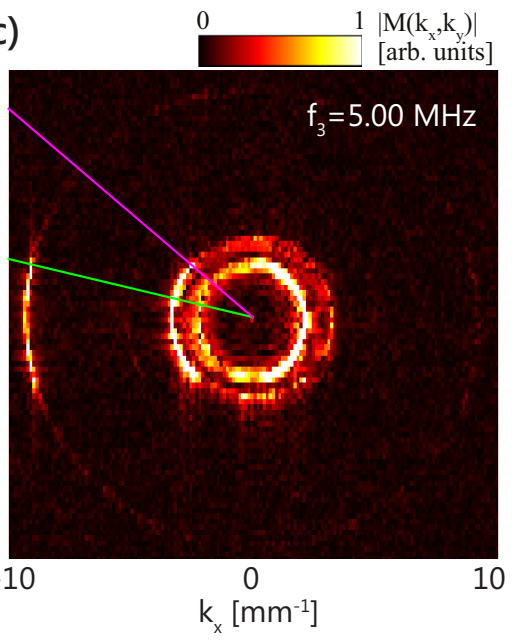

FIG. 7. Fourier domain representation of the wave fields from the experiment at frequencies of (a) 5.050, (b) 5.025, and (c) 5.000 MHz. As in Fig. 2, the inner ring corresponds to the excited $S_{2 b}$ mode and the outer circular segments show the reflected $S_{0}$ and $S_{1}$ modes. The green and magenta lines show the maximum possible angles of reflection for the $S_{0}$ and $S_{1}$ modes, respectively, calculated from Snell's law using the experimentally obtained $k$ values.

$13.4^{\circ}$ for the three frequencies. The angular range of negative reflection falls close to that expected for both modes, and the magnitude of the reflected $S_{1}$ mode appears relatively uniform within this range. In order to compare the experimental and simulated spectra, the axes in the experimental spectra should be multiplied by 0.578 to account for the smaller plate thickness. Taking into account the scaling factor, we find general agreement between simulation [see Figs. 3(a)-3(c)] and experiment.

\section{CONCLUSIONS}

Backward-propagating waves can be used to manipulate wave fields in unusual and nonintuitive ways. Furthermore, backward-propagating elastic waves exist in very simple finite elastic structures such as rods and plates. Here we demonstrate broad-angle negative reflection at the free edge of a plate upon mode conversion between a backward- $\left(S_{2 b}\right)$ and a forward- $\left(S_{1}\right)$ propagating wave. Such mode conversion is quite efficient near the zero-group-velocity point, due to the fact that the mode shapes for a given wave vector are nearly identical for the two modes in this region while the energy of the two modes propagates in opposite directions. This allows a reflected $S_{1}$ mode with a group velocity away from the edge to nearly satisfy the edge boundary conditions for an incident $S_{2 b}$ mode. We also demonstrated through both simulation and experiment that negative reflection leads to focusing of the $S_{1}$ field in the plate, and that the position of this focus can be controlled through the excitation frequency. The focus approaches the excitation position as the wave vectors of the $S_{1}$ and $S_{2 b}$ modes come close together near the zero-group-velocity point. Negative reflection of waves in elastic waveguides may find application in the nondestructive characterization of these structures. It may also serve usefully in the development of new acoustic devices including lenses, resonators, and filters.

\section{ACKNOWLEDGMENTS}

I.A.V. and C.G. acknowledge support from ongoing research programs of the FWF Austrian Science Fund, Project No. P 26162-N20; T.W. M. and D.M.S. gratefully acknowledge the support of this research by the National Science Foundation under Grant No. CMMI 1335426.
[1] H. Lamb, On group velocity, Proc. London Math. Soc. s2-1, 473 (1904).

[2] R. A. Shelby, D. R. Smith, and S. Schultz, Experimental verification of a negative index of refraction, Science 292, 77 (2001).

[3] A. A. Houck, J. B. Brock, and I. L. Chuang, Experimental Observation of a Left-Handed Material That Obeys Snell's Law, Phys. Rev. Lett. 90, 137401 (2003).

[4] N. Fang, D. Xi, J. Xu, M. Ambati, W. Srituravanich, C. Sun, and $\mathrm{X}$. Zhang, Ultrasonic metamaterials with negative modulus, Nat. Mater. 5, 452 (2006).
[5] J. Li, and C. T. Chan, Double-negative acoustic metamaterial, Phys. Rev. E 70, 055602(R) (2004).

[6] S. Zhang, L. Yin, and N. Fang, Focusing Ultrasound with an Acoustic Metamaterial Network, Phys. Rev. Lett. 102, 194301 (2009).

[7] J. B. Pendry, Negative Refraction Makes a Perfect Lens, Phys. Rev. Lett. 85, 3966 (2000).

[8] S. A. Cummer, Simulated causal subwavelength focusing by a negative refractive index slab, Appl. Phys. Lett. 82, 1503 (2003).

[9] A. Sukhovich, B. Merheb, K. Muralidharan, J. O. Vasseur, Y. Pennec, P. A. Deymier, and J. H. Page. Experimental and 
Theoretical Evidence for Subwavelength Imaging in Phononic Crystals. Phys. Rev. Lett. 102, 154301 (2009).

[10] N. Fang, H. Lee, C. Sun, and X. Zhang, Sub-diffraction-limited optical imaging with a silver superlens, Science 308, 534 (2005).

[11] M. Notomi, Theory of light propagation in strongly modulated photonic crystals: Refractionlike behavior in the vicinity of the photonic band gap, Phys. Rev. B 62, 10696 (2000).

[12] E. Cubukcu, K. Aydin, E. Ozbay, S. Foteinopoulou, and C. M. Soukoulis, Electromagnetic waves: Negative refraction by photonic crystals, Nature (London) 423, 604 (2003).

[13] S. Yang, J. H. Page, Z. Liu, M. L. Cowan, C. T. Chan, and P. Sheng, Focusing of Sound in a 3D Phononic Crystal, Phys. Rev. Lett. 93, 024301 (2004).

[14] M. Ibanescu, S. G. Johnson, D. Roundy, C. Luo, Y. Fink, and J. D. Joannopoulos, Anomalous Dispersion Relations by Symmetry Breaking in Axially Uniform Waveguides, Phys. Rev. Lett. 92, 063903 (2004).

[15] A. H. Meitzler, Backward-wave transmission of stress pulses in elastic cylinders and plates, J. Acoust. Soc. Am. 38, 835 (1965).

[16] I. Tolstoy and E. Usdin, Wave propagation in elastic plates: Low and high mode dispersion, J. Acoust. Soc. Am. 29, 37 (1957).

[17] P. L. Marston, Negative group velocity Lamb waves on plates and applications to the scattering of sound by shells, J. Acoust. Soc. Am. 113, 2659 (2003).

[18] A. A. Maznev and A. G. Every, Surface acoustic waves with negative group velocity in a thin film structure on silicon, Appl. Phys. Lett. 95, 011903 (2009).

[19] A. L. Shuvalov and O. Poncelet, On the backward Lamb waves near thickness resonances in anistropic plates, Int. J. Solids Struct. 45, 3430 (2008).

[20] C. Prada, D. Clorennec, and D. Royer, Local vibration of an elastic plate and zero-group velocity Lamb modes, J. Acoust. Soc. Am. 124, 203 (2008).

[21] S. Mezil, F. Bruno, S. Raetz, J. Laurent, D. Royer, and C. Prada, Investigation of interfacial stiffnesses of a tri-layer using zerogroup velocity Lamb modes, J. Acoust. Soc. Am, 138, 3202 (2015).
[22] S. D. Holland and D. E. Chimenti, Air-coupled acoustic imaging with zero-group-velocity Lamb modes, Appl. Phys. Lett. 83, 2704 (2003).

[23] D. Clorennec, C. Prada, and D. Royer, Local and noncontact measurements of bulk acoustic wave velocities in thin isotropic plates and shells using zero group velocity Lamb modes, J. Appl. Phys. 101, 034908 (2007).

[24] D. Clorennec, C. Prada, and D. Royer, Laser ultrasonic inspection of plates using zero-group velocity Lamb modes, IEEE Trans. Ultrason. Ferroelectr. Freq. Control 57, 1125 (2010).

[25] F. D. Philippe, T. W. Murray, and C. Prada, Focusing on plates: Controlling guided waves using negative refraction, Sci. Rep. 5, 11112 (2015).

[26] S. Bramhavar, C. Prada, A. A. Maznev, A. G. Every, T. B. Norris, and T. W. Murray, Negative refraction and focusing of elastic Lamb waves at an interface, Phys. Rev. B 83, 014106 (2011).

[27] M. Germano, A. Alippi, A. Bettucci, and G. Mancuso, Anomalous and negative reflection of Lamb waves in mode conversion, Phys. Rev. B 85, 012102 (2012).

[28] G. S. He, Optical phase conjugation: Principles techniques, and applications, Prog. Quantum Electron. 26, 131 (2002).

[29] J.D. Achenbach, Wave Propagation in Elastic Solids (NorthHolland, Amsterdam, 1980).

[30] P. J. Torvik, Reflection of wave trains in semi-infinite plates, J. Acoust. Soc. Am. 41, 346 (1967).

[31] B. Morvan, N. Wilkie-Chancellier, H. Duflo, A. Tinel, and J. Duclos, Lamb wave reflection at the free edge of a plate, J. Acoust. Soc. Am. 113, 1417 (2003).

[32] R. D. Gregory and I. Gladwell, The reflection of a symmetric Rayleigh-Lamb wave at the fixed or free edge of a plate, J. Elasticity 13, 185 (1983).

[33] A. Gunawan and S. Hirose, Reflection of obliquely incident guided waves by an edge of a plate, Mater. Trans. 48, 1236 (2007).

[34] S. Santhanam and R. Demirli, Reflection of Lamb waves obliquely incident on the free edge of a plate, Ultrasonics $\mathbf{5 3}$, 271 (2013).

[35] R. Zhu, X. N. Liu, and G. L. Huang, Study of anomalous wave propagation and reflection in semi-infinite elastic metamaterials, Wave Motion 55, 73 (2015). 